\title{
Caractérisation acoustique de la cavitation en conduites
}

\section{Determination of acoustic characteristics of pipe cavitation}

\author{
Jean-Pierre De Loof et Daniel Leducq
}

Société Bertin \& Cie

\section{Introduction}

La cavitation apparaît au sein d'un écoulement de liquide lorsque la pression statique locale devient inférieure à une "pression critique" voisine de la tension de vapeur saturante du liquide à la température de l'écoulement. Ce phénomène représente une gène importante, voire un danger pour l'utilisateur par ses manifestations : baisse de rendement, érosion, bruits, vibrations. Il n'est pas toujours possible d'éviter la cavitation, aussi a-t-on cherché à se protéger de ses manifestations qui apparaissent de prime abord comme les plus dangereuses, en particulier de l'érosion. Le bruit et les vibrations engendrés ont moins retenu l'attention des chercheurs, bien que les nuisances et fatigues mécaniques qu'ils provoquent peuvent devenir causes de gênes prohibitives, voire dangereuses.

Parmi les travaux publiés sur le problème des bruits et vibrations produits par la cavitation, on peut distinguer deux catégories :

- Un certain nombre de chercheurs, depuis Raighleigh, se sont penchés sur le problème théorique de l'évolution d'une bulle de cavitation : formation, vibrations et déformations, implosions, entraînement dans l'écoulement.

- Le passage du cas d'une bulle individuelle au cas réel de nuages de bulles étant particulièrement complexe, en particulier à cause du nombre élevé de paramètres régissant le phénomène, on trouve dans la littérature une autre catégorie de travaux portant sur des cas particuliers d'application précise :

bruit de la cavitation débutante, en vue de l'utilisation de sa mesure comme moyen de détection (on s'intéresse alors au bruit haute fréquence : quelques centaines de $\mathrm{kHz}$ ) ;
- bruits et vibrations induits sur des hélices et appendices de navires;

recherches de formules empiriques s'appliquant à des organes de circuits hydrauliques, données par le fabricant : celles-ci sont en général très approchées et ont un domaine d'application très restreint.

Il apparaissait donc nécessaire de reprendre une étude plus systématique du phénomène de cavitation sous l'angle du bruit et des vibrations qu'il engendre aux basses fréquences, pour mieux pouvoir les prévoir, et partant s'en protéger.

Nous avons conçu et réalisé à cet effet une boucle d'essai à températures, pressions et débits variables, où la cavitation est déclenchée par une singularité qui est un diaphragme.

Sur le plan expérimental on a mesuré simultanément, au droit du diaphragme, à l'amont immédiat et à l'aval de ce dernier, les niveaux et spectres de pression dans la bande audible par capteurs placés à la paroi.

On a relevé également la température de l'eau et la vitesse de l'écoulement. Les phénomènes ont été suivis visuellement et enregistrés à l'ultra-cinéma, ce qui a permis de mettre en évidence la création d'ondes de choc se déplaçant à l'aval de la poche cavitante.

Les résultats ont été analysés et interprétés dans l'optique de la recherche de l'influence des différents paramètres gouvernant l'écoulement, en vue d'agir sur eux pour réduire ou supprimer les bruits et vibrations engendrés par la cavitation.

On s'est aperçu que la poche de cavitation d'une part et l'onde de choc se créant à l'aval de celle-ci d'autre part, séparaient l'écoulement en plusieurs régions bien distinctes où les phénomènes étaient nettement différenciés. L'analyse spectrale met en évidence des bruits 
basse fréquence de niveau très important, fonction à la fois de la vitesse et de la figure de cavitation. Les phénomènes analysés se présentent donc comme ceux liés à la propagation d'un choc dans une conduite. Nous n'avons $\mathrm{pu}$, dans le cadre de cette étude, considérée comme exploratoire, exploiter au maximum les informations recueillies. Les résultats semblent intéressants et mériteraient, à notre avis, d'être complétés et exploités plus en détail.

\section{Conduite de la recherche}

\section{Description du moyen d'essai}

Une "boucle de cavitation" a été spécialement conçue pour cette étude, où il fallait pouvoir obtenir des écoulements à haute vitesse, à pression et température variables, avec peu de bruit propre de l'installation ramené au niveau de la veine d'essai.

L'ensemble "boucle de cavitation" est constitué de deux cuve cylindriques reliées par la veine d'essai. Un groupe moto-pompe complète le circuit et permet la circulation du fluide (voir figure 1).

La veine d'essai a un diamètre de $80 \mathrm{~mm}$ et une longueur de $2 \mathrm{~m}$. Elle est réalisée en altuglass pour permettre l'observation des phénomènes. Elle est composée de deux tronçons d'égale longueur entre lesquels on peut interposer des diaphragmes donnant différents rapports de contraction. Dans la configuration étudiée ici, nous avons adopté un rapport de $1 / 2$.
La variation de vitesse, dans une plage allant de $3 \mathrm{~m} / \mathrm{s}$ à $10 \mathrm{~m} / \mathrm{s}$, est obtenue en agissant à la fois sur le niveau de l'eau dans les cuves amont et aval, et sur la pression de volume d'air emprisonné.

Ces cuves d'un volume de $2 \mathrm{~m}^{3}$, jouent également un rôle d'amortisseur pour les éventuels à-coups de pression et de débit engendrés dans le circuit d'alimentation. Elles jouent également un rôle de filtre acoustique.

Le maintien d'un niveau d'eau constant de valeur déterminée dans chacune des cuves est assuré en agissant sur une vanne guillotine dont le but est de créer une perte de charge variable à l'entrée de la cuve amont. Les perturbations créées par cette vanne dans l'écoulement sont amorties en quasi totalité par un "doigt de gant" en tôle perforée dont le fond est tapissé d'une mousse plastique de $5 \mathrm{~cm}$ d'épaisseur.

Afin d'étendre la plage de réglage des débits, il a été réalisé un système de by-pass entre les canalisations d'arrivée et de retour du fluide. Ce by-pass est équipé d'une vanne de réglage à manchon souple.

\section{Contrôle de la température et de la pression}

Pour assurer l'exploration en température dans une plage $15^{\circ} \mathrm{C}, 40^{\circ} \mathrm{C}$, on a disposé dans la partie inférieure de la cuve amont une canne chauffante de $15 \mathrm{~kW}$ de puissance maximale, et dans la cuve aval un serpentin en acier inox de $27 \mathrm{~mm}$ de diamètre intérieur et $96 \mathrm{~m}$ de longueur déployée. Il a été ainsi possible d'explorer toute la plage de température envisagée.

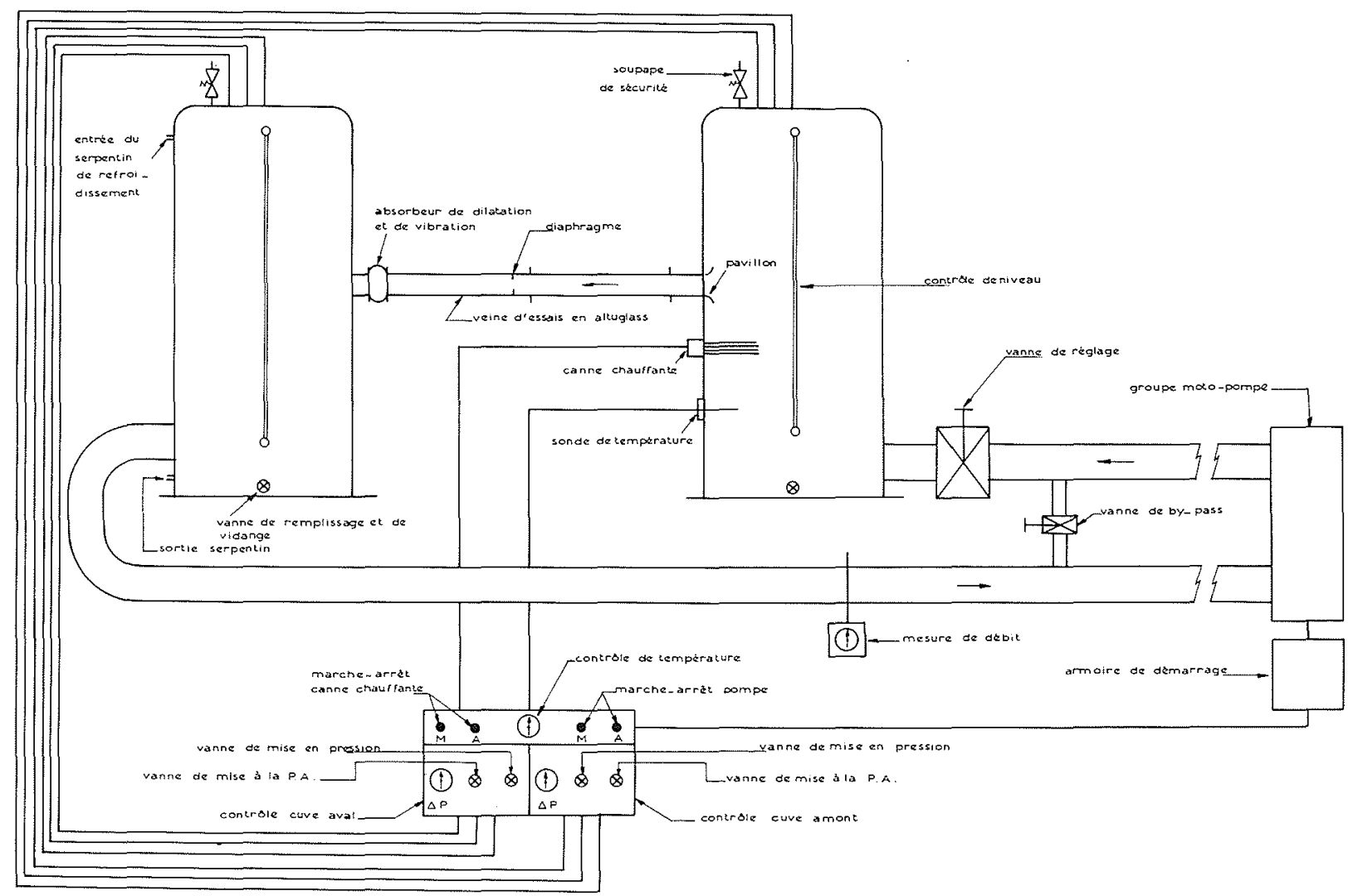

Figure 1 - Schéma de la boucle de cavitation. 
Le contrôle de la pression s'est effectué en raccordant la partie supérieure des cuves à l'air comprimé de la ville par un premier système de tuyauteries et à la mise à l'air libre ou en dépression par un second système (cf. figure 1). On est ainsi arrivé à pressuriser les cuves jusqu'à 3 bars absolus sans difficultés particulières. On est également descendu inversement à 0,5 bar dans la cuve aval dans la configuration de dépression maximale.

\section{Description du fonctionnement}

Le déroulement d'un essai type s'effectue de la façon suivante :

- les deux cuves sont au départ à la pression atmosphérique ou pressurisées au $\Delta_{p}$ recherché. Toutes les vannes de mise à l'air libre et en pression sont fermées,

- le démarrage s'effectue avec les deux vannes de réglage de débit ouvertes.

Le système de régulation thermique est mis en œuvre en fonction du point de consigne recherché.

On obtient une mise en pression de la cuve amont et une mise en dépression de la cuve aval par rapport aux pressions initiales. Les niveaux se stabilisent dans les deux cuves avec la mise en régime de la pompe.

On a placé dans la veine d'essai un diaphragme $\phi 60 \mathrm{~mm}$ cionnant un rapport de section de 1/2. Par réglage du débit à l'aide des deux vannes décrites cidessus, on fait varier le débit et on observe l'évolution des figures de cavitation lorsque la vitesse croit :

- bulles isolées avec crépitement,

- filets instables, claquement,

- poche stable, attachée au diaphragme, bruit intense.

Les régimes de mesure recherchés l'ont été en fonction de ces observations des figures de cavitation.

\section{Description des moyens de mesure}

\section{Mesure de la vitesse}

Elle a été réalisée à l'aide d'un peigne de pitots placé dans le conduit de retour entre la cuve aval et la pompe, l'intégration du profil de vitesses ainsi obtenu permettant de connaître le débit.

\section{Détermination des pressions d'entrée et sortie de la veine d'essai}

Un manomètre placé en haut de chaque cuve permet de mesurer la pression de l'air au-dessus du niveau de l'eau. Le niveau de l'eau, par rapport à l'axe de la veine est mesuré sur un tube gradué placé en parallèle sur chaque cuve. La colonne d'eau ainsi mesurée et convertie en pression est ajoutée à la pression d'air pour obtenir la pression $p_{\infty}$ amont et $p_{\infty}$ aval.

\section{Mesure de la température}

La cuve amont est équipée à cet effet d'une sonde à dilatation de liquide "téléthermomètre".

\section{Mesure de la teneur en air}

La teneur en air de l'eau est un paramètre très im- portant dans l'étude du phénomène qui nous intéresse. Elle intervient d'ailleurs essentiellement par la présence d'une quantité d'air non dissout sous une forme de bulles, qui sont autant de "germes" jouant un rôle de catalyseurs au départ, mais aussi un rôle amortisseur ultérieurement, au stade de la cavitation pleinement développée. C'est pourquoi nous avons cherché à mesurer cette population en germes. Nous avons tenté d'utiliser pour cela une sonde optique fonctionnant d'après le principe suivant : il s'agit d'une fibre optique recourbée dans sa partie en contact avec le milieu à mesurer ; à une extrémité on envoie un faisceau lumineux ; si la sonde est en contact avec une bulle de gaz, le faisceau revient normalement jusqu'au récepteur, il y a réflexion totale. Si la partie sensible est en contact avec l'eau, le faisceau diffuse à l'extérieur et le récepteur ne reçoit plus rien. On a réalisé ainsi un compteur de bulles. On obtient un signal en créneaux "tout ou rien" qui, traité, permet d'obtenir le taux de vide et le spectre de taille des germes. Vu la faible teneur en air à l'amont du diaphragme, les résultats obtenus n'ont malheureusement pu être utilisés dans le cadre de cette recherche.

Dans le cas présent, nous n'avons pu obtenir que des estimations très approximatives de la teneur en air, en supposant l'équilibre entre air et eau dans la cuve amont et que l'eau était saturée en air aux conditions de pression et température des essais effectués. Cette lacune n'enlève cependant pas le caractère de généralité des résultats qualitatifs obtenus ici.

\section{Mesures de bruit}

Elles ont été effectuées par capteurs piezzo-résistifs placés affleurant la paroi. Ces capteurs ont l'avantage d'être insensibles aux vibrations. Ils ne sont pas étanches cependant. On les a rendus étanches en recouvrant leur surface sensible avec une résine, puis l'ensemble a été placé dans un cylindre de plexiglas dont la face en contact avec l'écoulement a été mise au rayon de courbure de la veine d'essai, et réétalonnée.

Deux capteurs ont été utilisés, il s'agit de capteurs :

ENTRANS DEVICES EPI 080

- étendue de mesure $350 \mathrm{mb}$

- $\phi$ extérieur $2 \mathrm{~mm}$

- sensibilité $0,25 \mathrm{mV} / \mathrm{mb}$

- alimentation $15 \mathrm{~V}$

- résonance $125 \mathrm{kHz}$

- seuil de mesure $3,5 \mathrm{mb}$

possibilité de compensation par contre pression.

Deux séries de mesures ont été effectuées : une série avec les deux capteurs placés de part et d'autre du diaphragme et une série avec un capteur au droit de la zone cavitante et un capteur à l'entrée de la cuve aval. Ces capteurs passant le continu nous ont permis d'obtenir la pression statique locale, l'envoi d'une contre-pression permettant la mesure des fluctuations avec plus de sensibilité.

\section{Contrôle de la figure de cavitation}

Ce contrôle a pu être effectué en filmant à la caméra ordinaire et à 1000 images-seconde une même figure 
de cavitation. Le film à 1000 images-seconde a ensuite été analysé image par image. On a pu y identifier des structures analogues à des ondes de choc à l'aval immédiat de la poche de cavitation et estimer la célérité de celles-ci.

En dehors de ces enregistrements cinématographiques, les observations à ce niveau sont restées visuelles et phénoménologiques.

\section{Programme d'essai}

Présentation générale

On a dans un premier temps un seul diaphragme pour 3 vitesses différentes, 2 pressions et 2 températures différentes.
Pour chaque configuration, on a enregistré les niveaux et spectres de bruit en 3 points :

- à l'amont de la singularité,

- à l'aval immédiat de celle-ci,

- à l'aval de la singularité.

On a filmé à la caméra ultra-rapide l'évolution des figures de cavitation pour une vitesse de $6,8 \mathrm{~m} / \mathrm{s}$ à $15^{\circ} \mathrm{C}$ et sans surpression.

La plage d'enregistrement de bruit est celle pouvant être considérée comme industriellement gênante ou dangereuse soit sensiblement de $40 \mathrm{~Hz}$ à $20 \mathrm{kHz}$.

La suite des essais effectués est indiquée dans le tableau ci-après:

\begin{tabular}{|c|c|c|c|l|c|}
\hline $\begin{array}{c}\text { Essai } \\
n^{\circ}\end{array}$ & $\begin{array}{c}n^{\circ} \\
\text { capteur }\end{array}$ & $\begin{array}{c}\text { vitesse } \\
\text { moyenne }\end{array}$ & $\begin{array}{c}\Delta_{p} \\
\text { surpression }\end{array}$ & $\begin{array}{c}\text { observations } \\
\text { visuelles }\end{array}$ & $t^{\circ} \mathrm{C}$ \\
\hline 7 & $1-2$ & $7,8 \mathrm{~m} / \mathrm{s}$ & 0 & poche peu développée & 14,5 \\
8 & $1-2$ & $8,1 \mathrm{~m} / \mathrm{s}$ & 0 & $\begin{array}{l}\text { poche développée } \\
\text { flashes }\end{array}$ & 15 \\
9 & $1-2$ & $8,1 \mathrm{~m} / \mathrm{s}$ & $3 \times 10^{4} \mathrm{~Pa}$ & rien & 13,5 \\
10 & $1-3$ & $4,2 \mathrm{~m} / \mathrm{s}$ & 0 & flashes & 13,5 \\
11 & $1-3$ & $6,8 \mathrm{~m} / \mathrm{s}$ & 0 & poche peu développée & 13,5 \\
12 & $1-3$ & $7,8 \mathrm{~m} / \mathrm{s}$ & 0 & poche développée & 13,5 \\
13 & $1-3$ & $8,1 \mathrm{~m} / \mathrm{s}$ & 0 & rien & 14 \\
14 & $1-3$ & $4,2 \mathrm{~m} / \mathrm{s}$ & $3 \times 10^{4} \mathrm{~Pa}$ & 14 \\
15 & $1-3$ & $6,8 \mathrm{~m} / \mathrm{s}$ & $3 \times 10^{4} \mathrm{~Pa}$ & rien (crépitements audibles) & 14,5 \\
16 & $1-3$ & $7,8 \mathrm{~m} / \mathrm{s}$ & $3 \times 10^{4} \mathrm{~Pa}$ & flashes & 15 \\
17 & $1-3$ & $8,2 \mathrm{~m} / \mathrm{s}$ & $3 \times 10^{4} \mathrm{~Pa}$ & flashes & 30 \\
18 & $1-3$ & $4,2 \mathrm{~m} / \mathrm{s}$ & 0 & rien & 31 \\
19 & $1-3$ & $6,8 \mathrm{~m} / \mathrm{s}$ & 0 & flashes importants & 31,5 \\
20 & $1-3$ & $7,8 \mathrm{~m} / \mathrm{s}$ & 0 & poche développée & 3 \\
21 & $1-3$ & $8,1 \mathrm{~m} / \mathrm{s}$ & 0 & poche très développée & \\
\hline
\end{tabular}

\section{Analyse des résultats de mesure}

\section{Traitement des données}

Les signaux des capteurs de pression ont été enregistrés sur bande magnétique analogique, puis numérisés. Une première analyse spectrale sur le signal analogique ayant montré que la majeure partie de l'énergie se trouvait en basse fréquence ( $\mathrm{fc}<500 \mathrm{~Hz}$ ) nous avons décidé de faire l'analyse sur deux domaines de fréquences :

- une première analyse entre 0 et $1600 \mathrm{~Hz}$ sur le signal numérisé et filtré par un filtre passe-bas numérique,

- une seconde analyse entre 1600 et $16000 \mathrm{~Hz}$ sur le signal numérisé et filtré par un filtre passe-haut numérique.

Dans les deux cas, pour chaque essai, nous avons sorti l'autospectre de chaque signal, l'intercorrélation et la cohérence des signaux sortant des 2 capteurs mis en jeu.

Nous avons également examiné image par image le film obtenu à 1000 images-seconde dans le cas de l'écoulement à $6,8 \mathrm{~m} / \mathrm{s}, 15^{\circ} \mathrm{C}$ et sans surpression.

Les résultats dans la gamme $1600-16000 \mathrm{~Hz}$, recoupant des observations déjà connues sur le bruit de cavi- tation dans cette gamme de fréquences, nous avons effectué notre analyse sur la partie basse-fréquence du spectre, où l'on a mis en évidence des phénomènes qui peuvent s'interpréter assez aisément sur le plan physique, mais qui n'avaient jusqu'à présent, à notre connaissance, fait l'objet de recherches systématiques.

\section{Caractéristiques générales des résultats obtenus}

Les résultats obtenus par les 3 capteurs P1, P2, P3 affleurant à la paroi au droit de la source, immédiatement à l'amont de celle-ci et à l'aval, ont mis en évidence entre 10 et $2000 \mathrm{~Hz}$ tout d'abord une rupture d'impédance au niveau de la source entraînant une dissymétrie entre propagation amont et aval.

Les niveaux spectraux rencontrés immédiatement à l'amont de la singularité sont d'un ordre de grandeur inférieur à ceux mesurés immédiatement à l'aval et l'allure des spectres est différente (on met en évidence à l'amont du diaphragme des résonances qui ne se retrouvent pas à l'aval (cf. Fig. 2))

L'étude des niveaux globaux et des spectres au droit de la source montre également des différences avec les résultats obtenus en monophasique. 


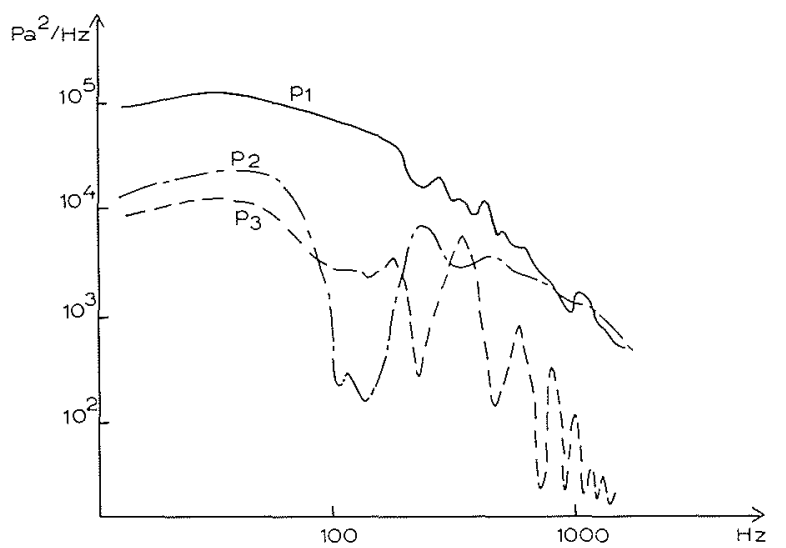

Figure 2 - Comparaison des niveaux spectraux aux différents points de mesure $(\mathrm{V} \approx 8 \mathrm{~m} / \mathrm{s})$.

Dans le cas d'écoulements monophasiques, sans cavitation, les composantes de la perturbation de pression reçues par le capteur sont de 3 ordres :

- le bruit de proximité créé par la couche limite : son niveau global moyen (écart type des fluctuations) sera pour l'exemple de l'écoulement à $8 \mathrm{~m} / \mathrm{s}$ que nous avons testé, $300 \mathrm{~Pa}$ environ, la fréquence caractéristique du spectre (largeur de la bande à niveau constant) sera de $200 \mathrm{~Hz}$ (cf. réf. 1, 2);

- la perturbation locale de pression créée par 1'introduction du diaphragme qui est liée au $\Delta_{p}$ singulier créé par ce dernier. Dans notre cas, on peut considérer que $\Delta_{p}$ vaut une dynamique, soit $32000 \mathrm{~Pa}$ pour $8 \mathrm{~m} / \mathrm{s}$. L'écart type maximum, au recollement du jet "sortant" du diaphragme, est de l'ordre de $0,1 \Delta_{p}$ (cf. réf. 3), soit $3200 \mathrm{~Pa}$. La fréquence de coupure qui correspond à un Strouhal de 0,2 à 0,3 calculés sur la différence des diamètres, vaut sensiblement $100 \mathrm{~Hz}$;

- la "source acoustique" (cf. réf. 4) qui a un niveau dix fois plus faible que la précédente, soit $320 \mathrm{~Pa}$ et une fréquence caractéristique de $10 \mathrm{~Hz}$.

Les signaux recueillis en présence de cavitation donnent les niveaux sensiblement plus élevés : toujours pour la même vitesse, écart type de l'ordre de $10^{4}$ pascals et fréquence de coupure située vers $200 \mathrm{~Hz}$. Le niveau spectral basse fréquence est augmenté d'environ $10 \mathrm{~dB}$. La cavitation modifie donc sensiblement les caractéristiques de la source.

En résumé, on peut considérer que la cavitation a un rôle d'amplificateur à large bande des phénomènes hydrodynamiques locaux, auxquels viennent se superposer la rupture d'impédance signalée ci-dessus liée à la brusque variation de compressibilité du fluide.

\section{Résultats d'analyse spectrale}

\section{Capteur P1 au droit de la poche cavitante}

On constate dans la gamme $0-200 \mathrm{~Hz}$ des niveaux très élevés, les allures des auto-spectres ne suivant pas, comme signalé ci-dessus, les lois classiques du bruit enregistré à la paroi d'une conduite contenant un fluide en écoulement turbulent, ni celles gouvernant la source de pression d'une singularité en conduite.

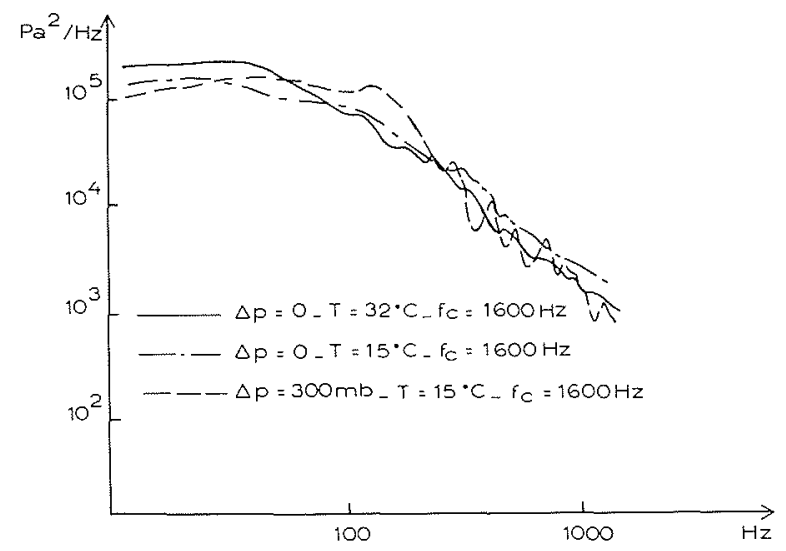

Figure 3 - Variation du niveau spectral au capteur $P_{1}$ en fonction des conditions thermodynamiques $(\mathrm{V} \cong 8 \mathrm{~m} / \mathrm{s})$

On constate ainsi, à $4,2 \mathrm{~m} / \mathrm{s}$, que le niveau sans cavitation apparente qui est de $10^{4} \mathrm{~Pa}^{2} / \mathrm{Hz}$ à $15^{\circ}$ et sans surpression augmente à la fois lorsque la pression augmente et lorsque la température augmente. Dans les trois cas, la fréquence de coupure à partir de laquelle le spectre décroît de façon continue est de $100 \mathrm{~Hz}$.

Dans tous les autres cas, le niveau croît avec la vitesse à pression et température données et, pour une même vitesse, celui-ci décroît quand la pression augmente et croît quand la température augmente, donc croît avec l'intensité de cavitation (cf. Fig. 3).

La fréquence de coupure suit, elle, l'évolution inverse : elle croît quand la pression augmente et décroît quand la température augmente.

Ceci confirme bien que le phénomène enregistré est gouverné essentiellement par la figure de cavitation. En effet, à vitesse donnée, on peut considérer la "poche" de cavitation comme une cavité déformable, dont la fréquence moyenne est fonction inverse de sa longueur caractéristique ; plus la pression est élevée, plus cette longueur est faible, donc plus la fréquence est élevée. Inversement, plus la température est élevée, plus le seuil de cavitation est atteint rapidement, plus la longueur caractéristique est élevée et plus la fréquence est faible.

\section{Capteur P2 à l'entrée de la cuve aval}

Les enregistrements en P2 ont été effectués dans le cas de cavitation développée ou fortement développée. Les spectres trouvés présentent une similitude de forme frappante.

Le niveau spectral moyen est divisé cette fois sensiblement par 50 et 100 par rapport aux niveaux trouvés en P1.

Par ailleurs, on constate unc coupure dans le spectre, comme si celui-ci était la juxtaposition de 2 spectres correspondant à deux phénomènes différents. La coupure se trouve entre $90 \mathrm{~Hz}$ et $200 \mathrm{~Hz}$ par $7,8 \mathrm{~m} / \mathrm{s}$, elle se déplace entre $50 \mathrm{~Hz}$ et $150 \mathrm{~Hz}$ à $8,1 \mathrm{~m} / \mathrm{s}$, mais on retrouve les mêmes valeurs lorsqu'à vitesse égale à $8,1 \mathrm{~m} / \mathrm{s}$, on augmente la pression de façon à retrouver ces conditions de cavitation analogues à celles trouvées pour $7,8 \mathrm{~m} / \mathrm{s}$. Ceci indique encore, comme pour P1, 
que ces fréquences caractéristiques sont directement liées à la figure de cavitation. Au-delà de $100 \mathrm{~Hz}$ à $200 \mathrm{~Hz}$ on constate une décroissance continue du spectre.

\section{Capteur P3 à l'amont immédiat du diaphragme}

Il est remarquable de constater avec les spectres obtenus que P3 ne semble essentiellement influencé que par l'écoulement et l'information acoustique lui venant de l'amont ou les vibrations du diaphragme lui-même, l'ensemble diaphragme + poche de cavitation jouant en quelque sorte le rôle d'écran acoustique. Les niveaux ne suivent plus du tout l'évolution observée pour $\mathrm{P} 1$, on constate une série d'harmoniques dont le fondamental se situe vers $60 \mathrm{~Hz}$ qui doit correspondre à un accrochage d'un élément amont du circuit qui n'a pu être identifié avec certitude dans le cadre de cette étude.

\section{Intercorrélations et cohérences}

Comme on pouvait s'y attendre à l'examen des autospectres, qui sont d'allure très différente d'un capteur à l'autre, les intercorrélations et cohérences sont de niveau faible généralement (cf. Fig. 4, 5).

On note cependant entre $\mathrm{P} 1$ et $\mathrm{P} 2$ un plateau de cohérence de niveau non négligeable correspondant avec le plateau BF du spectre de P2 avant la coupure BF. Ceci indique clairement que jusqu'à la première coupure, le spectre obtenu en P2 est gouverné essentiellement par la poche de cavitation.

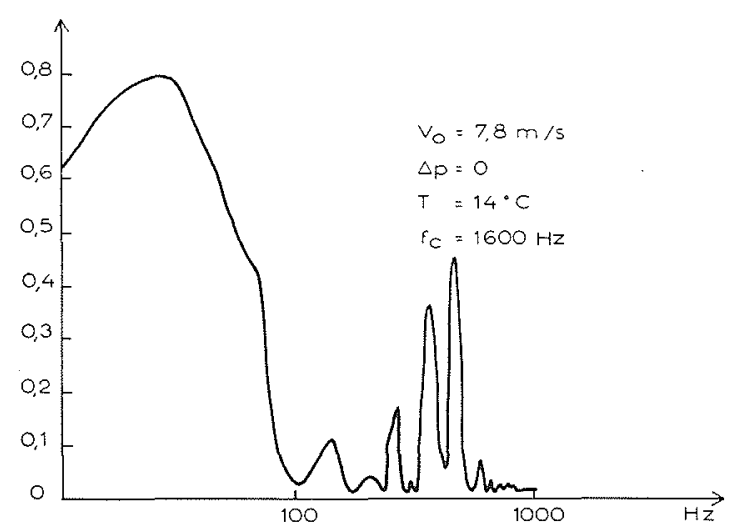

Figure 4 - Cohérence $P_{1} / P_{2}$

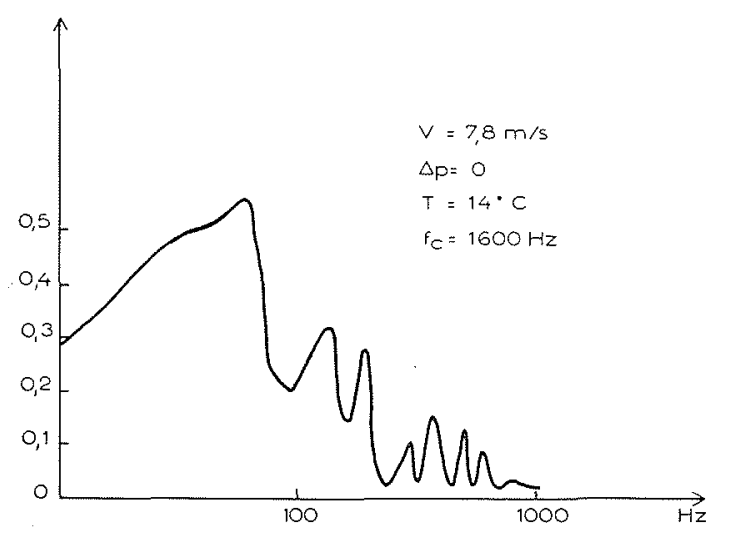

Figure $5-$ Cohérence $\mathrm{P}_{1} / \mathrm{P}_{2}$
En ce qui concerne $\mathrm{P} 1$ et $\mathrm{P} 3$, les niveaux sont relativement faibles également. Il faut noter toutefois des pics plus ou moins prononcés sur les harmoniques décelées dans le spectre de P3, ce qui veut dire qu'une petite part du signal acoustique reçu en P3 est transmise vers l'aval et traduit l'efficacité de la "poche" de cavitation en tant qu'écran.

On notera également aux fortes vitesses et à pression et température "normales" un plateau de cohérence voisin de 0,5 dans la gamme de 30 à $60 \mathrm{~Hz}$. Ce plateau disparaît à la fois lorsque la pression augmente et lorsque la température augmente, donc lorsque la pression statique locale augmente au droit de la poche cavitante. Ceci pourrait signifier que les phénomènes mis en jeu font intervenir des interactions entre le fluide et le diaphragme, interactions variant suivant la "rigidité" relative du milieu fluide. Mais ce n'est qu'une hypothèse qui reste entièrement à vérifier.

\section{Interprétation des enregistrements cinématographiques}

L'examen au ralenti du film à 1000 images-seconde pris dans le cas de l'écoulement à $6,8 \mathrm{~m} / \mathrm{s}$ a permis de visualiser des structures quasi-périodiques (zones plus foncées, normales à l'écoulement) se déplaçant de l'amont vers l'aval immédiatement derrière la poche de cavitation.

L'examen image par image du film montre que ces structures, faisant penser aux ondes se développant derrière un réacteur à gaz chauds, se propagent par trains de 3 ou 4 dans le cas étudié et disparaissent après avoir parcouru l'équivalent de 1 à 2 diamètres de veine, elles sont distantes entre-elles de $1,5^{-2}$ à $3 \times 10^{-2} \mathrm{~m}$ et leur vitesse de propagation est de l'ordre de $10 \mathrm{~m} / \mathrm{s}$ à $30 \mathrm{~m} / \mathrm{s}$.

Notons qu'à cette vitesse, il faut entre 25 et $75 \mathrm{~ms}$ pour que "l'onde" atteigne le capteur placé à l'entrée de la cuve aval. Or, la "période" de l'intercorrélation réalisée dans des conditions voisines (à $7,8 \mathrm{~m} / \mathrm{s}$ ) est de l'ordre de $40 \mathrm{~ms}$ (cf. Fig. 6) ce qui est parfaitement
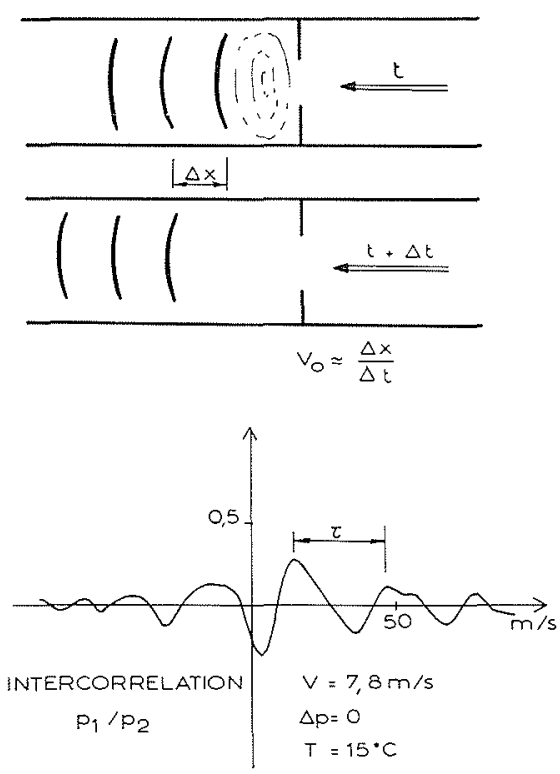

Figure 6 - Visualisation - Interprétation 
compatible avec l'hypothèse envisagée à partir de cet examen visuel d'une onde "de choc" se propageant derrière la poche de cavitation.

\section{Conclusions}

Cette étude constitue la "phase exploratoire" d'une étude plus poussée, destinée à identifier la cavitation en tant que source de bruit et vibrations en conduite et à qualifier cette source, aussi ne peut-elle être que limitée dans ses conclusions.

Une première analyse des phénomènes abordés nous a permis de mettre en évidence déjà quelques caractéristiques principales de ceux-ci :

- L'énergie se trouve concentrée essentiellement dans les basses, voire les très basses fréquences.

- Il y a rupture d'impédance acoustique au droit de la figure de cavitation et la transmission acoustique n'est pas identique entre l'amont et l'aval.

- Les phénomènes ne sont pas linéaires au voisinage immédiat de la poche de cavitation où semblent naître et se propager des "ondes de chocs" dont la célérité est de l'ordre de la vitesse locale de l'écoulement.

- Le "modèle acoustique" d'une telle source serait alors celui d'un piston soumis à des oscillations amorties et se déplaçant au sein de l'écoulement. Cette image relativement simple demande cependant à être étayée et confirmée par de nombreux essais complémentaires plus systématiques et une analyse plus orientée dans le sens de cette modélisation.

\section{Références}

[1]CLINCH J.M. - Measurements of the wall pressure field at the surface of a smoothwalled pipe containing turbulent water flow. U.S.V. 1969, vol. 9, pp. 398-419.

[2] NICOLET J.B., SOUQUET J., MARTIN J.M. - Etudes et essais en vue de caractériser les sources des vibrations aérodynamiques. Rapport de fin de contrat D.G.R.S.T., 1972.

[3]GIBERT R.J. - Etude expérimentale de deux singularités d'un circuit. Note C.E.A. N 1735.

[4] HENRY R. - Mise en évidence et analyse des instationnarités élastiques dans les écoulements internes. Thèse d'Etat Faculté d'Orsay.

\section{Discussion}

\begin{abstract}
Le Président. - Je remercie M. LEDUCQ et j'ouvre la discussion.

M. GUITON. - En ce qui concerne les dernières images du film présenté, je voudrais rappeler que M. HUGUENIN, du Laboratoire des Arts et Métiers, a fait, il y a une dizaine d'années des expérimentations de cet ordre sur la cavitation ou les diaphragmes et il avait trouvé aussi ces tranches alternativement sombres et claires, qu'il avait appelées "cavitation en piles d'assiettes", mais sans donner d'explications.
\end{abstract}

M. LEDUCQ. - M. HUGUENIN a effectivement publié un article sur la cavitation en conduite dans La Houille Blanche il $\mathrm{y}$ a près de 10 ans. Mais je n'avais pas souvenir de cette observation. L'interprétation que j'ai proposée vient de ma formation d'aérodynamicien et de l'analogie des images du film avec les visualisations de gaz chauds en sortie de réacteurs.

M. BERHAULT. - Les spectres de cavitation présentent un maximum d'énergie dans des bandes de fréquences beaucoup plus élevées - à partir de 15 ou $18 \mathrm{kHz}$ - que celles présentées ici. Comment expliquez-vous cette différence ?

M. LEDUCQ. - Je me suis volontairement placé dans un domaine qui n'est pas habituellement abordé dans l'étude de la cavitation : celui de son influence sur le plan "acoustique". Les recherches antérieures portaient sur le domaine hautes fréquences $(\sim 100 \mathrm{kHz})$ en vue de la détection de la cavitation. Je me suis placé dans le domaine des nuisances créées par la cavitation en basses fréquences. Le niveau spectral y est notablement augmenté et la cavitation crée des "accrochages" gênants, excitant des résonances de structures (cf. chants des hélices) par les impulsions locales de pression à large bande qu'elle engendre.

M. BONNIN, - Le caractère impulsionnel du bruit de cavitation, mentionné par l'auteur, et correspondant aux évè- nements successifs que sont les implosions de bulles, reçoit sa confirmation dans le chant des hélices qui vient d'être mentionné, et aussi dans l'expérience suivante.

Lors d'essais de cavitation en sodium (non publiés), nous avons observé la superposition, au bruit d'écoulement à spectre étalé, d'un spectre de raies ; l'interprétation a pu l'attribuer à l'excitation de parties mécaniques par ces impulsions.

L'auteur interprète par ailleurs l'écoulement cavitant à l'aval du diaphragme comme une série d'ondes de choc. Cette interprétation peut appeler quelques réserves : elle pourrait en particulier être liée au système étudié.

Enfin l'interprétation d'un effet de température attribué à une variation du nombre de germes me paraît très discutable. En fait, les faibles variations de température permises par l'installation dans les essais rapportés (moins de $3 \%$ de la température critique du liquide, à un niveau thermique inférieur à $50 \%$ de cette température) ne permettent pas de mettre en évidence les véritables effets thermiques en cavitation.

Je voudrais mentionner que, lors d'essais à température très voisine de la température critique ( 90 à $95 \%$ de cette dernière), on a pu observer une cavitation parfaitement exempte de bruit.

M. LECOFFRE. - Des expérimentations récemment réalisées au Delft Hydraulic Laboratory ont montré que le bruit émis par une vanne dépend grandement de la teneur en germes du liquide.

Le liquide d'essais utilisé était de l'eau à température ordinaire, saturée en air à pression atmosphérique ou fortement dégazée, dans laquelle étaient ou non injectés des germes artificiels formés de micro-bulles.

La cavitation, produite sur une vanne fermée de diaphragmes multiples, était du type "cavitation de mélange" ou de jet, donc très influencée par la teneur en germes. 
On a pu montrer que la saturation en air dissous du liquide n'a pas d'influence directe sur la valeur du $\sigma$ de début de cavitation $\left(\sigma_{\mathfrak{i}}\right)$.

Par contre, la variation de teneur en air libre peut faire varier la valeur de $\sigma_{i}$ dans des proportions considérables. Pour une telle vanne, fonctionnant sous une chute de pression de l'ordre de $1 \mathrm{bar}$, on a constaté une variation de $\sigma_{i}$ allant du simple au double selon que des germes artificiels étaient ou non injectés.

Par ailleurs, en ce qui concerne les méthodes de mesure de germes, certaines, dites directes, comptent les germes de cavita- tion en créant effectivement des bulles de vapeur (système Venturi de Delft ou Neyrtec). D'autres, dites indirectes, mesurent la dimension géométrique des particules ou microbulles présentes dans le liquide.

On a pu ainsi montrer qu'un nombre très faible des particules contenues dans l'eau est susceptible de donner naissance à des bulles de vapeur. Le rapport du nombre de germes efficaces au nombre de germes total, essentiellement des particules solides, peut être de l'ordre de $1 / 1.000 .000$.

Le Président. - Je remercie M. LEDUCQ. 